\title{
Applications of the Sylvester operator in the space of slice semi-regular functions
}

https://doi.org/10.1515/conop-2020-0001

Received November 6, 2019; accepted December 16, 2019

\begin{abstract}
In this paper we apply the results obtained in [3] to establish some outcomes of the study of the behaviour of a class of linear operators, which include the Sylvester ones, acting on slice semi-regular functions. We first present a detailed study of the kernel of the linear operator $\mathcal{L}_{f, g}$ (when not trivial), showing that it has dimension 2 if exactly one between $f$ and $g$ is a zero divisor, and it has dimension 3 if both $f$ and $g$ are zero divisors. Afterwards, we deepen the analysis of the behaviour of the $A$-product, giving a complete classification of the cases when the functions $f_{v}, g_{v}$ and $f_{v} \wedge g_{v}$ are linearly dependent and obtaining, as a by-product, a necessary and sufficient condition on the functions $f$ and $g$ in order their *-product is slice-preserving. At last, we give an Embry-type result which classifies the functions $f$ and $g$ such that for any function $h$ commuting with $f+g$ and $f \star g$, we have that $h$ commutes with $f$ and $g$, too.
\end{abstract}

Keywords: Slice-regular functions, Sylvester equation, *-product of slice-regular functions, semi-regular functions, idempotent functions, Embry's Theorem

MSC: Primary 30G35; secondary: 39B42, 47A56

\section{Introduction}

The aim of this paper is to exploit the powerfulness of Sylvester operators in the context of slice regularity, thus providing a series of outcomes, some of which quite unexpected, that deal with properties of commutation of semi-regular functions on axially symmetric domains contained in the skew algebra of quaternions. For a self-contained introduction to the subject of slice-regular and semi-regular functions see $[9,11,13]$, while a systematic study of Sylvester operators in this setting can be found in [3].

After shortly recalling the main definitions, in Section 3 the results obtained in [3] allow us to show that the kernel of the linear operator $\mathcal{L}_{f, g}(\chi)=f{ }^{\star} \chi{ }^{\star} g$ has dimension 2 if and only if exactly one between $f$ and $g$ is a zero divisor, while it has dimension 3 if and only if $f$ and $g$ are both zero divisors. As a consequence, we are able to give a complete description of the set of all solutions of the equation $\mathcal{L}_{f, g}(\chi)=\mathfrak{b}$ also when $\mathcal{L}_{f, g}$ is not an isomorphism.

We then turn to study the behaviour of the A product of two semi-regular functions $f$ and $g$ defined on $\Omega$. If the domain $\Omega$ contains real points, it is easy to show that if $f_{v}$ and $g_{v}$ are linearly independent, then also $f_{v}, g_{v}$ and $f_{v} \AA g_{v}$ are (see Proposition 4.1). The case when $\Omega$ has not real points is much more complicated: in particular when $f_{v}$ and $g_{v}$ are linearly independent, Corollary 4.4 states necessary and sufficient conditions in order there exist $\alpha$, $\beta$ slice preserving functions defined on $\Omega$ such that $\alpha f_{v}+\beta g_{v}+f_{v} \wedge$ A $g_{v} \equiv 0$. In Proposition 4.5 we use this result to give a complete characterization of the functions $f, g$ whose *-product is slice preserving.

Altavilla Amedeo: Dipartimento di Ingegneria Industriale e Scienze Matematiche, Università Politecnica delle Marche, Via Brecce Bianche, 60131, Ancona, Italy, E-mail: altavilla@dipmat.univpm.it, GNSAGA of INdAM

*Corresponding Author: Chiara de Fabritiis: Dipartimento di Ingegneria Industriale e Scienze Matematiche, Università Politecnica delle Marche, Via Brecce Bianche, 60131, Ancona, Italia, E-mail: fabritiis@dipmat.univpm.it, GNSAGA of INdAM 
Finally, Section 5 contains an analogous of Embry's theorem in the case of semi-regular functions: we are able to classify the couples of semi-regular functions $f$, $g$ defined on $\Omega$ such that any semi-regular function $h$ defined on $\Omega$ which commutes with $f+g$ and $f^{\star} g$ also commutes with $f$ and $g$. In particular, if the domain $\Omega$ contains real points, we have that the only case in which this does not happen is when $g=f^{c}$, which implies that $f_{v}$ and $g_{v}$ are linearly dependent, and thus gives that the functions $f$ and $g$ commute, though it is not sure that $h$ commutes with $f$ and $g$.

\section{Definitions and preliminary results}

We denote by $\mathbb{H}$ the skew algebra of quaternions and by $\mathbb{S} \subset \mathbb{H}$ the set of imaginary units $\mathbb{S}:=\left\{q \in \mathbb{H} \mid q^{2}=\right.$ -1 . Any quaternion $q \in \mathbb{H}$ can be written as $q=x+I y$, where $I \in \mathbb{S}, x, y \in \mathbb{R}$ and $y \geq 0$; the quantities $x$ and $y$ are always uniquely determined, while $I$ is unique if and only if $y \neq 0$. A set $\Omega \subset \mathbb{H}$ is said to be axially symmetric if for any $q=x+I y \in \Omega$, then $x+J y$ belongs to $\Omega$ for any $J \in \mathbb{S}$; an axially symmetric domain $\Omega$ is said to be slice if $\Omega \cap \mathbb{R} \neq \emptyset$, is said to be product otherwise.

If $\Omega$ is an axially symmetric domain, a function $f: \Omega \rightarrow \mathbb{H}$ is said to be slice regular if, for any $q=x+I y \in$ $\Omega, f(q)=F_{1}(x, y)+I F_{2}(x, y)$ (that is, $f$ is quaternionic-left-affine with respect to the imaginary unit), $\left(F_{1}, F_{2}\right)$ is an even-odd couple of quaternionic-valued function with respect to $y$ and $F_{1}, F_{2}$ satisfy the usual CauchyRiemann equations. In the sequel, we will denote the family of slice regular functions defined on $\Omega$ by $\mathcal{R}(\Omega)$. The pointwise sum obviously preserves regularity, whilst this is not true for the point-wise product. However, a natural notion of product, inspired by the power series case, can be introduced: given $f$ and $g$ two slice regular functions, if $f(x+I y)=F_{1}(x, y)+I F_{2}(x, y)$ and $g(x+I y)=G_{1}(x, y)+I G_{2}(x, y)$, then we define the *-product as

$$
\left(f^{\star} g\right)(x+I y):=\left(F_{1}(x, y) G_{1}(x, y)-F_{2}(x, y) G_{2}(x, y)\right)+I\left(F_{1}(x, y) G_{2}(x, y)+F_{2}(x, y) G_{2}(x, y)\right) .
$$

With this product the set $\mathcal{R}(\Omega)$ becomes an associative algebra; being $F_{m}$ and $G_{n}$ quaternionic valued (for $m, n=1,2$ ), the *-product is clearly non-commutative. However if $F_{m}$ and $G_{n}$ take value in a fixed complex line $\mathbb{C}_{I}:=\operatorname{Span}_{\mathbb{R}}(1, I) \subset \mathbb{H}$, then $f^{\star} g=g{ }^{\star} f$; moreover, if $F_{1}$ and $F_{2}$ take real values, then, for any $g$ we have $f{ }^{\star} g=g \star f$. This two properties motivate the following definition.

Definition 2.1. Let $\Omega \subset \mathbb{H}$ be an axially symmetric domain, $f: \Omega \rightarrow \mathbb{H}$ be a slice regular function and $J \in \mathbb{S}$ be an imaginary unit. If, for any $q=x+I y \in \Omega$, the function $f(x+I y)=F_{1}(x, y)+I F_{2}(x, y)$ is such that

- $\quad F_{1}$ and $F_{2}$ take values in $\mathbb{C}_{J}$ (equivalently if $f\left(\Omega \cap \mathbb{C}_{J}\right) \subset \mathbb{C}_{J}$ ), then we say that $f$ is $\mathbb{C}_{J}$-preserving;

- $\quad F_{1}$ and $F_{2}$ take values in $\mathbb{R}$ (equivalently if $f\left(\Omega \cap \mathbb{C}_{J}\right) \subset \mathbb{C}_{J}$, for all $J \in \mathbb{S}$ ), then we say that $f$ is slice preserving.

The set of $\mathbb{C}_{J}$-preserving functions is denoted by $\mathcal{R}_{J}(\Omega)$, while the set of slice preserving functions by $\mathcal{R}_{\mathbb{R}}(\Omega)$. For a detailed study of the features of slice preserving and one-slice preserving functions, see [2].

It is easily seen that the set of slice regular functions on $\Omega$ is the center of $\mathcal{R}(\Omega)$. Thanks to a result by Colombo, Gonzalez-Cervantes and Sabadini [8] (see also [10] for a generalization), fixed an orthonormal basis $(1, i, j, k)$ of $\mathbb{H}$, it is possible to write any slice regular function $f \in \mathcal{R}(\Omega)$ as

$$
f=f_{0}+f_{v}=f_{0}+f_{1} i+f_{2} j+f_{3} k,
$$

where $f_{\ell} \in \mathcal{R}_{\mathbb{R}}(\Omega)$ for all $\ell=0,1,2,3$ are uniquely determined. If we define the regular conjugate of a slice regular function $f=f_{0}+f_{v}$ as $f^{c}=f_{0}-f_{v}$, then $f$ is slice preserving if and only if $f=\left(f+f^{c}\right) / 2=f_{0}$. If $I$ is any imaginary unit, we have that $f$ is $\mathbb{C}_{I}$-preserving if and only if, for any orthogonal basis $(1, I, J, K) \subset \mathbb{H}$, we have that $f=f_{0}+f_{1} I$.

The strength of this representation is expressed in the following formula which provides a formal relationship with the theory of real quaternions: if $f=f_{0}+f_{v}, g=g_{0}+g_{v}$ are slice regular functions, then

$$
f \star g=f_{0} g_{0}-\left\langle f_{v}, g_{v}\right\rangle_{\star}+f_{0} g_{v}+g_{0} f_{v}+f_{v} \wedge g_{v},
$$


where $\langle,\rangle_{\star}$ and $\AA_{A}$ are the formal generalizations of the usual scalar and vector product, i.e.

$$
\langle f, g\rangle_{\star}:=f_{0} g_{0}+f_{1} g_{1}+f_{2} g_{2}+f_{3} g_{3}, \quad f_{v} \text { A } g_{v}:=\left(f_{2} g_{3}-f_{3} g_{2}\right) i+\left(f_{3} g_{1}-f_{1} g_{3}\right) j+\left(f_{1} g_{2}-f_{2} g_{1}\right) k,
$$

provided $(1, i, j, k)$ is a positive orthonormal basis of $\mathbb{H}$.

The idea of exploiting the above formula in order to obtain new analytic and algebraic results in slice regularity was introduced and developed in [1].

By working with this formal generalization, one quickly realizes the needs of invertible elements. This can be done by allowing poles and working with slice semi-regular functions. For a self-contained introduction to the topic of semi-regular function we refer to [12, 13]. In particular, given any $f \in \mathcal{R}(\Omega)$, we define the symmetrized function of $f$ as the slice preserving function defined by $f^{s}:=f^{\star} f^{c}=\langle f, f\rangle$ * and the regular inverse as $f^{-\star}:=\left(f^{s}\right)^{-1} f^{c}$.

Let $\Omega$ be an axially symmetric domain and $p=x+I y \in \Omega \backslash \mathbb{R}$. Ghiloni, Perotti and Stoppato [13] proved that any $f \in \mathcal{R}\left(\Omega \backslash \mathbb{S}_{p}\right)$ can be written around $\mathbb{S}_{p}:=\{x+J y: J \in \mathbb{S}\}$ as

$$
f(q)=\sum_{n \in \mathbb{Z}}(q-p)^{\star n} b_{n},
$$

with $b_{n} \in \mathbb{H}$, for any $n \in \mathbb{Z}$. The point $p$ is called a removable singularity if $f$ extends to a slice regular function in a circular open set containing $\mathbb{S}_{p}$. If it is not a removable singularity, the point $p$ is said to be a pole for $f$ if there exists an $n_{0} \geq 0$ such that $b_{n}=0$ for all $n<n_{0}$. If $p$ is neither a removable singularity nor a pole, then it is called an essential singularity for $f$.

Definition 2.2. A function $f$ is said to be slice semi-regular in a nonempty circular domain $\Omega$, if there exists a circular open subset $\widetilde{\Omega} \subseteq \Omega$ such that $f \in \mathcal{R}(\widetilde{\Omega})$ and such that each point of $\Omega \backslash \widetilde{\Omega}$ is either a pole or a removable singularity for $f$. The set of slice semi-regular functions on $\Omega$ will be denoted as $\mathcal{R} \mathcal{N}(\Omega)$; the sets of slice preserving and of $\mathbb{C}_{I}$-preserving (for some $I \in \mathbb{S}$ ) semi-regular functions on $\Omega$ as $\mathcal{R \mathcal { M }}_{\mathbb{R}}(\Omega)$ and $\mathcal{R \mathcal { M }}_{I}(\Omega)$, respectively.

It is not difficult to prove that, for any axially symmetric domain $\Omega$, then $\left(\mathcal{R \mathcal { N }}_{\mathbb{R}}(\Omega),+,{ }^{\star}\right)$ is a field and $\mathcal{R} \mathcal{N}(\Omega)$ is an associative algebra which can be seen as a 4-dimensional vector space over $\mathcal{R \mathcal { N }}_{\mathbb{R}}(\Omega)$ (see [3]). Moreover if $\Omega$ has no real points, i.e., it is a product domain, it is possible to find regular functions defined on $\Omega$ that are non-trivial idempotents with respect to the ${ }^{*}$-product.

Definition 2.3. Let $q=q_{0}+q_{v} \in \mathbb{H} \backslash \mathbb{R}$. We define the slice preserving regular function $\mathcal{J}: \mathbb{H} \backslash \mathbb{R} \rightarrow \mathbb{H}$ as $\mathcal{J}(q)=\frac{q_{v}}{\left|q_{v}\right|}$.

Notice that $\mathcal{J} \star \mathcal{J}=\mathcal{J} \mathcal{J}=\mathcal{J}^{2} \equiv-1$. Starting from the function $\mathcal{J}$ it is possible to define the following two "fundamental" idempotents (see [4, Definition 2.3]).

Definition 2.4. Let $q=q_{0}+q_{v} \in \mathbb{H} \backslash \mathbb{R}$ and fix $i \in \mathbb{S}$. We define the slice regular functions $\ell^{+, i}, \ell^{-, i}: \mathbb{H} \backslash \mathbb{R} \rightarrow \mathbb{H}$ as

$$
\ell^{+, i}(q):=\frac{1-\mathcal{J} i}{2}, \quad \ell^{-, i}(q):=\frac{1+\mathcal{J} i}{2} .
$$

A straightforward computation shows that these functions satisfy the following equalities

$$
\left(\ell^{+, i}\right)^{c}=\ell^{-, i}, \quad\left(\ell^{+, i}\right)^{S}=\left(\ell^{-, i}\right)^{S}=\ell^{+, i} \star \ell^{-, i} \equiv 0 \quad \text { and } \quad \ell^{+, i} \star \ell^{+, i}=\ell^{+, i} .
$$

In [3, Proposition 2.13] we proved that $f \in \mathcal{R} \mathcal{N}(\Omega) \backslash\{0,1\}$ is an idempotent if and only if it belongs to $\mathcal{R}(\Omega)$ and is a zero divisor (i.e. $f^{s} \equiv 0$ ) such that $f_{0} \equiv \frac{1}{2}$ (and thus $f_{v}^{s} \equiv-\frac{1}{4}$ ).

In the same paper we introduced the following two operators.

Definition 2.5. Let $f, g \in \mathcal{R} \mathcal{N}(\Omega) \backslash\{0\}$. We denote by $\mathcal{L}_{f, g}, \mathcal{S}_{f, g}: \mathcal{R} \mathcal{N}(\Omega) \rightarrow \mathcal{R} \mathcal{N}(\Omega)$ the $\mathcal{R \mathcal { M }}_{\mathbb{R}}(\Omega)$-linear operators given by

$$
\mathcal{L}_{f, g}(\chi):=f^{\star} \chi^{\star} g, \quad \mathcal{S}_{f, g}(\chi):=f^{\star} \chi+\chi^{\star} g
$$


the second being called Sylvester operator.

An introduction to Sylvester operators is contained in [6,7] and [14], to which we direct the interested reader for further references. The kernel of the Sylvester operator is deeply linked with the concept of equivalency between regular functions. In particular we recall the following definition and result.

Definition 2.6. Let $f, g \in \mathcal{R \mathcal { N }}(\Omega)$. We say that $f$ and $g$ are equivalent and write $f \simeq g$ if there exists a *invertible $h \in \mathcal{R} \mathcal{N}(\Omega)$, such that

$$
f=h^{-\star} g^{\star} h \text {. }
$$

In [3] we proved the following result, which gives as a direct by-product that all idempotents different from 0 and 1 are equivalent (since they both have "real part" equal to $\frac{1}{2}$ and the symmetrized functions of their "vector parts" are both equal to $-\frac{1}{4}$ ).

Theorem 2.7. Let $f, g \in \mathcal{R \mathcal { N }}(\Omega) \backslash \mathcal{R \mathcal { M }}_{\mathbb{R}}(\Omega)$. The following facts are equivalent

- $\quad r k\left(\mathcal{S}_{f, g}\right)=2$;

- $f \simeq-g$;

- $f_{0}=-g_{0}$ and $f_{v}^{S}=g_{v}^{s}$

- $\operatorname{ker}\left(\mathcal{S}_{f, g}\right)$ contains at least an invertible element in $\mathcal{R N}(\Omega)$.

\section{The behaviour of the operator $\mathcal{L}_{f, g}$}

We start by recalling the following result stating when the operator $\mathcal{L}_{f, g}$ is an isomorphism (see [3]).

Proposition 3.1. Let $f, g \in \mathcal{R} \mathcal{N}(\Omega) \backslash\{0\}$.

1. The operator $\mathcal{L}_{f, g}$ is an isomorphism if and only if neither $f$ nor $g$ are zero divisors.

2. If $\mathcal{L}_{f, g}$ is an isomorphism, then for any $\mathfrak{b} \in \mathcal{R M}(\Omega)$ the equation $\mathcal{L}_{f, g}(\chi)=\mathfrak{b}$ has the unique solution $\chi=$ $f^{-\star} \star \mathfrak{b}^{\star} g^{-\star}$.

3. If $\mathcal{L}_{f, g}$ is an isomorphism, then the solution of $\mathcal{L}_{f, g}(\chi)=\mathfrak{b}$ belongs to $\mathcal{R}(\Omega)$ for any $\mathfrak{b} \in \mathcal{R}(\Omega)$ if and only if $f$ and $g$ are never vanishing.

We now turn to the study of the operator $\mathcal{L}_{f, g}$ when at least one between $f$ and $g$ is a zero divisor. Next proposition completely settles the description of its kernel, including the computation of its dimension, deepening the content of [3, Proposition 3.5].

Proposition 3.2. Let $f, g \in \mathcal{R} \mathcal{N}(\Omega) \backslash\{0\}$.If exactly one between $f$ and $g$ is a zero divisor, then $\operatorname{dim} \operatorname{ker}\left(\mathcal{L}_{f, g}\right)=2$; if both $f$ and $g$ are zero divisors, then $\operatorname{dim} \operatorname{ker}\left(\mathcal{L}_{f, g}\right)=3$.

Proof. Thanks to [3, Proposition 2.14, Remark 2.16], if $f$ is a zero divisor, we can find a unitary quaternion $\eta$ such that $(f \eta)_{0} \not \equiv 0$ and $f=2(f \eta)_{0} \eta^{c \star} \rho_{f}$, where $\rho_{f}$ is an idempotent. Analogously if $g$ is a zero divisor, we can find a unitary quaternion $\delta$ such that $(g \delta)_{0} \not \equiv 0$ and $g=2(g \delta)_{0} \sigma_{g} \delta^{c}$, where $\sigma_{g}$ is an idempotent. Moreover, Proposition 9.1 in [3] ensures that any idempotent is conjugated to $\ell^{+, i}$, so that there exist $\varphi, \psi \in \mathcal{R} \mathcal{N}(\Omega)$ invertible such that

$$
\rho_{f}=\varphi \star \ell^{+, i} \star \varphi^{-\star} \quad \text { and } \quad \sigma_{g}=\psi \star \ell^{+, i} \star \psi^{-\star} .
$$

We start from the case in which exactly one between $f$ and $g$ (say $f$ ) is a zero divisor. Thus, being $g$ invertible, $\chi \in \operatorname{ker}\left(\mathcal{L}_{f, g}\right)$ if and only if $\chi \in \operatorname{ker}\left(\mathcal{L}_{f, 1}\right)$, that is $2(f \eta)_{0} \eta^{c} \star \rho_{f}{ }^{\star} \chi \equiv 0$. Since $(f \eta)_{0} \in \mathcal{R \mathcal { N }}_{\mathbb{R}}(\Omega) \backslash\{0\}$ and $\eta$ is a unitary quaternion, last equality is equivalent to $\rho_{f}{ }^{\star} \chi \equiv 0$. Acting now as in the proof of Proposition 9.2(1) in [3], we obtain that $\chi$ belongs to $\operatorname{ker}\left(\mathcal{L}_{f, g}\right)$ if and only if $\chi=\varphi \star \ell^{-, i \star}(\alpha+\beta j)$, with $\alpha, \beta \in \mathcal{R M}_{\mathbb{R}}(\Omega)$, 
which entails that the dimension of the kernel equals 2 . The proof of the specular case in which the only zero divisor is $g$ is performed with the same strategy.

We now turn to the case when both $f$ and $g$ are zero divisors. Reasoning as before we obtain that $\chi \in$ $\operatorname{ker}\left(\mathcal{L}_{f, g}\right)$ if and only if it belongs to the kernel of $\mathcal{L}_{\rho_{f}, \sigma_{g}}$. Thanks to Formula (3.1), this is equivalent to the fact that

$$
\ell^{+, i \star} \varphi^{-\star}{ }^{\star} \chi^{\star} \psi^{\star} \ell^{+, i} \equiv 0
$$

Proposition 9.2(3) in [3] guarantees that Equality (3.2) is satisfied if and only if

$$
\chi=\varphi^{\star}\left(\alpha \ell^{-, i}+\left(\beta_{1}+\beta_{2} i\right)^{\star} j\right){ }^{\star} \psi^{-\star},
$$

with $\alpha, \beta_{1}, \beta_{2} \in \mathcal{R} \mathcal{M}_{\mathbb{R}}(\Omega)$, thus proving that, in this case, $\operatorname{dim} \operatorname{ker}\left(\mathcal{L}_{f, g}\right)=3$.

As far as the image is concerned, Theorem 3.3 in [3] gives a necessary and sufficient condition in order that $\mathfrak{b}$ belongs to the range of $\mathcal{L}_{f, g}$ and its proof contains an explicit particular solution in all the singular cases. Combining this with the above proposition we obtain a description of the space of solutions of the equation $\mathcal{L}_{f, g}(\chi)=\mathfrak{b}$.

We set the notation as follows: if $f$ is a zero divisor, for a suitable unitary $\eta \in \mathbb{H}$, we write

$$
f=2(f \eta)_{0} \sigma_{f}^{\star} \eta^{c}
$$

with $\sigma_{f}=\varphi^{\star} \ell^{+, i} \star \varphi^{-\star}$ idempotent; if $g$ is a zero divisor, for a suitable unitary $\delta \in \mathbb{H}$, we write

$$
g=2(g \delta)_{0} \delta^{c} \rho_{g}
$$

with $\rho_{g}=\psi^{\star} \ell^{+, i \star} \psi^{-\star}$ idempotent. With this notation, Theorem 3.3 in [3] states that $\mathcal{L}_{f, g}(\chi)=\mathfrak{b}$ admits a solution if and only if $\mathfrak{b}=\sigma_{f} \star \mathfrak{b}$, if $f$ is a zero divisor, and $\mathfrak{b}=\mathfrak{b} \star \rho_{g}$, if $g$ is a zero divisor.

Proposition 3.3. Let $f, g, \mathfrak{b} \in \mathcal{R} \mathcal{N}(\Omega)$ be such that the operator $\mathcal{L}_{f, g}$ is singular and $\mathfrak{b}$ belongs to its range. If $f$ is a zero divisor and $g$ is not, the space of solutions of the equation $\mathcal{L}_{f, g}(\chi)=\mathfrak{b}$ is given by

$$
\left\{\left(2(f \eta)_{0}\right)^{-1} \eta^{\star} \mathfrak{b} \star g^{-\star}+\eta \star \varphi^{\star} \ell^{-, i} \star(\alpha+\beta j) \mid \alpha, \beta \in \mathcal{R \mathcal { M }}_{\mathbb{R}}(\Omega)\right\} ;
$$

if $g$ is a zero divisor and $f$ is not, the space of solutions of the equation $\mathcal{L}_{f, g}(\chi)=\mathfrak{b}$ is given by

$$
\left\{\left(2(g \delta)_{0}\right)^{-1} f^{-\star} \star \mathfrak{b} \star \delta+(\alpha+\beta j) \star \ell^{-, i} \star \psi^{-\star} \star \delta \mid \alpha, \beta \in \mathcal{R \mathcal { M }}_{\mathbb{R}}(\Omega)\right\} ;
$$

if both $f$ and $g$ are zero divisors, the space of solutions of the equation $\mathcal{L}_{f, g}(\chi)=\mathfrak{b}$ is given by

$$
\left\{\left[\left(2(f \eta)_{0}\right)^{-1} \eta\right] \star \mathfrak{b} \star\left[\left(2(g \delta)_{0}\right)^{-1} \delta\right]+\eta \star \varphi^{\star}\left[\alpha \star \ell^{-, i}+\left(\beta_{1}+\beta_{2} i\right) \star j\right] \star \psi^{-\star} \star \delta \mid \alpha, \beta_{1}, \beta_{2} \in \mathcal{R \mathcal { M }}_{\mathbb{R}}(\Omega)\right\} .
$$

Proof. We only deal with the case when $f$ is a zero divisor and $g$ is invertible, being the other ones completely analogous. The "particular" solution $\left(2(f \eta)_{0}\right)^{-1} \eta \star \mathfrak{b} \star g^{-\star}$ of the equation $\mathcal{L}_{f, g}(\chi)=\mathfrak{b}$ appears in the proof of Theorem 3.3 in [3], while the description of the kernel is obtained with the same technique used above in the proof of Proposition 3.2.

\section{Properties of the $A$-product}

In this section we analyze the behaviour of the $A$-product defined in [1]. When the domain $\Omega$ contains real points, a simple argument (see Proposition 4.1) shows that if $f_{v}$ and $g_{v}$ are not linearly dependent on $\mathcal{R M}_{\mathbb{R}}(\Omega)$, then $f_{v}, g_{v}, f_{V} \wedge g_{v}$ are linearly independent. When $\Omega$ is a product domain a very different situation can take place, that is $f_{v}, g_{v}$ and $f_{v} \wedge g_{v}$ can be linearly dependent. 
Proposition 4.1. Suppose $\Omega$ is a slice domain and take $f_{v}, g_{v}$ linearly independent on $\mathcal{R M}_{\mathbb{R}}(\Omega)$. Then $f_{v}, g_{v}, f_{v} \AA g_{v}$ are linearly independent on $\mathcal{R \mathcal { M }}_{\mathbb{R}}(\Omega)$.

Proof. If $\alpha f_{v}+\beta g_{v}+\gamma f_{v}$ ^ $g_{v} \equiv 0$, then by taking the $\langle,\rangle_{\star}$-product with $f_{v}$ ^ $g_{v}$ we obtain

$$
\gamma\left(f_{v} \AA g_{v}\right)^{s} \equiv 0 .
$$

Thus either $\gamma \equiv 0$ which entails $\alpha=\beta=0$, too, or $\left(f_{v} \wedge g_{v}\right)^{S} \equiv 0$. Since the domain $\Omega$ is slice, last equality yields that $f_{v} \wedge g_{v} \equiv 0$ and, thanks to [1, Proposition 2.10], this is a contradiction to the fact that $f_{v}, g_{v}$ are linearly independent.

As the case in which $\Omega$ is slice is completely understood, we now turn to the case in which $\Omega$ is a product domain. Let us consider $f_{v}$ and $g_{v}$ linearly independent and suppose that $f_{v}, g_{v}$ are linearly dependent, then there exists $\alpha, \beta$ and $\gamma$ in $\mathcal{R N}_{\mathbb{R}}(\Omega)$, not all identically zero, such that

$$
\alpha f_{v}+\beta g_{v}+\gamma f_{V} \AA g_{v} \equiv 0 .
$$

Since $f_{v}$ and $g_{v}$ are linearly independent, then $\gamma$ cannot be identically zero, so we are left to consider the following equality

$$
\alpha f_{v}+\beta g_{v}+f_{V} \text { ^ } g_{v} \equiv 0 .
$$

We start our study by considering a special case, namely when $f_{v}$ and $f_{v}$ A $g_{v}$ are linearly dependent, that is $\beta=0$ in Equality (4.1).

Theorem 4.2. Let $f_{v}$ and $g_{v}$ be linearly independent on $\mathcal{R M}_{\mathbb{R}}(\Omega)$. Then $f_{v}$ and $f_{v} A g_{v}$ are linearly dependent if and only if there exists $\alpha \in \mathcal{R} \mathcal{M}_{\mathbb{R}}(\Omega) \backslash\{0\}$ such that

1. $f_{v}^{S} \equiv 0$;

2. $\alpha^{2}+g_{v}^{S} \equiv 0$;

3. $\left\langle f_{v}, g_{v}\right\rangle_{\star} \equiv 0$;

4. for any $\delta \in \mathcal{R} \mathcal{N}(\Omega)$ with $\langle\delta, 1\rangle_{\star} \equiv 0$ and $\left\langle\delta, g_{v}\right\rangle_{\star} \equiv 0$, we have $f_{v}{ }^{\star} \delta^{\star}\left(\alpha-g_{v}\right) \equiv 0$.

Moreover, if $\alpha f_{v}+f_{v} \wedge g_{v} \equiv 0$ holds with $\alpha \in \mathcal{R} \mathcal{M}_{\mathbb{R}}(\Omega) \backslash\{0\}$, then there exists an invertible $h \in \mathcal{R} \mathcal{N}(\Omega)$ and $\lambda \in \mathcal{R \mathcal { M }}_{\mathbb{R}}(\Omega) \backslash\{0\}$, such that $\left.g_{v}=h^{-\star} \star \alpha \mathcal{J} i\right)^{\star} h$ and $f_{v}=h^{-\star} \star\left(\lambda \ell^{-, i \star} j\right){ }^{\star} h$ with $j \perp i$.

Proof. Since $f_{v}$ and $g_{v}$ are linearly independent, then $f_{v} \wedge g_{v} \not \equiv 0$, and thus $f_{v}$ and $f_{v} \wedge g_{v}$ are linearly dependent if and only if there exists $\alpha \in \mathcal{R \mathcal { M }}_{\mathbb{R}}(\Omega) \backslash\{0\}$ such that

$$
\alpha f_{v}+f_{v} \text { ^ } g_{v} \equiv 0 .
$$

By taking the "scalar products" of the two members of Equation (4.2) with both $f_{v}$ and $g_{v}$, we obtain that $f_{v}^{S} \equiv 0$ and $\left\langle f_{v}, g_{v}\right\rangle_{\star} \equiv 0$. The definition of the $\mathbb{A}$-product gives that Equation (4.2) is equivalent to

$$
\left(\alpha-g_{v}\right) \star f_{v}+f_{v} \star\left(\alpha+g_{v}\right) \equiv 0,
$$

and thus $f_{v}$ belongs to the kernel of the Sylvester operator $\mathcal{S}_{\alpha-g_{v}, \alpha+g_{v}}$ which coincides with the kernel of $\mathcal{S}_{1-g_{v} / \alpha, 1+g_{v} / \alpha}$. Thanks to Proposition 6.1 in [3], the fact that $f_{v} \not \equiv 0$ implies that $1+\frac{g_{v}^{s}}{\alpha^{2}} \equiv 0$, that is $\alpha^{2}+g_{v}^{S} \equiv 0$. Now, Theorem 8.1 in [3] guarantees that there exists $h \in \mathcal{R \mathcal { N }}(\Omega)$ invertible, such that $g_{v} / \alpha=h^{-\star}{ }^{\star}(J i){ }^{\star} h$, or equivalently $g_{v}=h^{-\star} \star(\alpha \mathcal{J} i) \star h$. By inserting last expression for $g_{v}$ in Equality (4.3), we obtain that $\tilde{f}_{v}:=h{ }^{\star} f_{V}{ }^{\star} h^{-\star}$ belongs to ker $\mathcal{S}_{1-\jmath i, 1+\jmath i}$. Formula (6.2) in [3], gives that the matrix associated to the linear operator $\mathcal{S}_{1-\jmath_{i, 1+\jmath_{i}}}$ is given by

$$
S_{1-\mathcal{J} i, 1+\mathcal{} i}=\left|\begin{array}{cccc}
2 & 0 & 0 & 0 \\
0 & 2 & 0 & 0 \\
0 & 0 & 2 & 2 \mathcal{J} \\
0 & 0 & -2 \mathcal{J} & 2
\end{array}\right|,
$$


whose kernel is spanned by $\ell^{-, i \star} j$. Thus $f_{v}=h^{-\star} \star\left(\lambda \ell^{-, i \star} j\right) \star h$ for a suitable $\lambda \in \mathcal{R} \mathcal{M}_{\mathbb{R}}(\Omega) \backslash\{0\}$.

Finally, notice that (4) is invariant by conjugation with $h$, so we are left to prove it in the case when $g_{v}=\alpha \mathcal{J} i$ and $f_{v}=\lambda \ell^{-, i} \star j$. Let $\delta=\delta_{2} j+\delta_{3} k$, with $\delta_{2}, \delta_{3} \in \mathcal{R} \mathcal{M}_{\mathbb{R}}(\Omega)$ and $k=i j$, then

$$
\begin{aligned}
f_{v} \star \delta & =\frac{\lambda}{2}(j+\partial k) \star\left(\delta_{2} j+\delta_{3} k\right)=\frac{\lambda}{2}\left(-\delta_{2}-\delta_{2} \partial i+\delta_{3} i-\delta_{3} \partial\right) \\
& =-\frac{\lambda}{2}\left(\delta_{2}(1+\partial i)+\delta_{3} \partial(1+\partial i)\right)=-\frac{\lambda}{2 \alpha}\left(\delta_{2}+\partial \delta_{3}\right)(\alpha+\alpha \mathcal{\partial} i)=-\frac{\lambda}{2 \alpha}\left(\delta_{2}+\partial \delta_{3}\right)\left(\alpha+g_{v}\right),
\end{aligned}
$$

which therefore entails $f_{v} \star \delta^{\star}\left(\alpha-g_{v}\right) \equiv 0$.

We now prove that (1)-(4) imply Formula (4.2). As all conditions are invariant under conjugation by invertible elements of $\mathcal{R} \mathcal{N}(\Omega)$, by (2) and Theorem 8.1 in [3] we can assume that $g_{v}=\alpha \mathcal{J} i$. Then, (3) gives that $f_{v}=f_{2} j+f_{3} k$ and hence (1) entails $f_{2}^{2}+f_{3}^{2} \equiv 0$. As $f_{v} \not \equiv 0$, we have that $f_{2} \not \equiv 0$ and thus $f_{3}^{2}=\left(\partial f_{2}\right)^{2}$ shows that either $f_{3} \equiv \mathcal{J} f_{2}$ or $f_{3} \equiv-\partial f_{2}$, that is either $f_{v}=f_{2}(j+\mathcal{J} k)$ or $f_{v}=f_{2}(j-\mathcal{J} k)$. In the second case, by taking $\delta=j$ we have $f_{v} \star j=-\frac{f_{2}}{\alpha}\left(\alpha-g_{v}\right)$ and so $f_{v}{ }^{\star} j\left(\alpha-g_{v}\right)=-\frac{f_{2}}{\alpha}\left(\alpha-g_{v}\right) \star\left(\alpha-g_{v}\right)$ which is not identically zero. Then the only possibility is given by $f_{v}=f_{2}(j+\jmath k)$ and a direct computation shows that Formula (4.2), which is also invariant under conjugation by invertible elements of $\mathcal{R} \mathcal{N}(\Omega)$, holds.

Corollary 4.3. Let $f_{v}$ and $g_{v}$ be linearly independent on $\mathcal{R} \mathcal{M}_{\mathbb{R}}(\Omega)$. Then $g_{v}$ and $f_{v} \wedge g_{v}$ are linearly dependent if and only if there exists $\beta \in \mathcal{R \mathcal { M }}_{\mathbb{R}}(\Omega) \backslash\{0\}$ such that

1. $g_{v}^{S} \equiv 0$;

2. $\beta^{2}+f_{v}^{s} \equiv 0$;

3. $\left\langle f_{v}, g_{v}\right\rangle_{\star} \equiv 0$;

4. for any $\eta \in \mathcal{R \mathcal { N }}(\Omega)$ with $\langle\eta, 1\rangle_{\star} \equiv 0$ and $\left\langle\eta, f_{v}\right\rangle_{\star} \equiv 0$, we have $\left(\beta-f_{v}\right)^{\star} \eta{ }^{\star} g_{v} \equiv 0$.

Moreover, if $\beta g_{v}+f_{v} \mathbb{A} g_{v} \equiv 0$ holds with $\beta \in \mathcal{R \mathcal { M }}_{\mathbb{R}}(\Omega) \backslash\{0\}$, then there exists an invertible $h \in \mathcal{R} \mathcal{M}(\Omega)$ and $\lambda \in \mathcal{R \mathcal { M }}_{\mathbb{R}}(\Omega) \backslash\{0\}$, such that $f_{v}=-h^{-\star} \star(\beta J i){ }^{\star} h$ and $g_{v}=h^{-\star} \star\left(\lambda \ell^{-, i \star} j\right){ }^{\star} h$.

Proof. The proof trivially follows from the fact that $\beta g_{v}+f_{v} \wedge g_{v} \equiv 0$ if and only if $-\beta g_{v}+g_{v}$ A $f_{v} \equiv 0$ and from Theorem 4.2.

A suitable modification of the functions involved in Theorem 4.2 allows us to classify all linearly independent functions $f_{v}, g_{v}$ such that $f_{v}, g_{v}$ and $f_{v}$ A $g_{v}$ are linearly dependent.

Corollary 4.4. Let $f_{v}$ and $g_{v}$ be linearly independent on $\mathcal{R \mathcal { M }}_{\mathbb{R}}(\Omega)$. Then $f_{v}, g_{v}$ and $f_{v} \AA g_{v}$ are linearly dependent if and only if there exist $\alpha, \beta \in \mathcal{R N}_{\mathbb{R}}(\Omega)$ such that

1. $\beta^{2}+f_{v}^{s} \equiv 0$;

2. $\alpha^{2}+g_{v}^{S} \equiv 0$;

3. $\left\langle f_{v}, g_{v}\right\rangle_{\star} \equiv \alpha \beta$;

4. (a) for any $\delta \in \mathcal{R \mathcal { N }}(\Omega)$ with $\langle\delta, 1\rangle_{\star} \equiv 0$ and $\left\langle\delta, g_{v}\right\rangle_{\star} \equiv 0$, we have $\left(\alpha f_{v}+\beta g_{v}\right)^{\star} \delta^{\star}\left(\alpha-g_{v}\right) \equiv 0$;

(b) for any $\eta \in \mathcal{R \mathcal { M }}(\Omega)$ with $\langle\eta, 1\rangle_{\star} \equiv 0$ and $\left\langle\eta, f_{v}\right\rangle_{\star} \equiv 0$, we have $\left(\beta-f_{v}\right)^{\star} \eta^{\star}\left(\alpha f_{v}+\beta g_{v}\right) \equiv 0$.

Moreover, if Formula (4.1) holds and $\alpha \neq \equiv$, there exists an invertible $h \in \mathcal{R N}(\Omega)$ and $\lambda \in \mathcal{R N}_{\mathbb{R}}(\Omega) \backslash\{0\}$, such that $g_{v}=h^{-\star} \star(\alpha \mathcal{J} i){ }^{\star} h$ and $f_{v}=h^{-\star} \star\left(-\beta \mathcal{J} i+\lambda \ell^{-, i \star} j\right){ }^{\star} h$.

Proof. Reasoning as in the proof of Theorem 4.2, we obtain that $f_{v}, g_{v}$ and $f_{v} \wedge g_{v}$ are linearly dependent if and only if there exists $\alpha, \beta \in \mathcal{R \mathcal { M }}_{\mathbb{R}}(\Omega)$ which are not both identically zero such that

$$
\alpha f_{v}+\beta g_{v}+f_{v} \AA g_{v} \equiv 0 .
$$

If exactly one between $\alpha$ and $\beta$ is identically zero, then we refer to Theorem 4.2 and Corollary 4.3. 
Hence we are left to deal with the case $\alpha \not \equiv 0$ and $\beta \not \equiv 0$. Since

$$
\left\langle f_{v}+\frac{\beta}{\alpha} g_{v}, g_{v}\right\rangle_{\star}=\left\langle f_{v}, g_{v}\right\rangle_{\star}+\frac{\beta}{\alpha} g_{v}^{s},
$$

then, thanks to (2), condition (3) can be written as $\left\langle f_{v}+\frac{\beta}{\alpha} g_{v}, g_{v}\right\rangle_{\star} \equiv 0$. As

$$
\left(f_{v}+\frac{\beta}{\alpha} g_{v}\right)^{s}=f_{v}^{s}+\frac{\beta^{2}}{\alpha^{2}} g_{v}^{s}+2 \frac{\beta}{\alpha}\left\langle f_{v}, g_{v}\right\rangle_{\star},
$$

then, thanks to (2) and (3), condition (1) is equivalent to $\left(f_{v}+\frac{\beta}{\alpha} g_{v}\right)^{s} \equiv 0$. Finally, $\alpha f_{v}+\beta g_{v}+f_{v}$ A $g_{v} \equiv 0$ is equivalent to $\alpha\left(f_{v}+\frac{\beta}{\alpha} g_{v}\right)+\left(f_{v}+\frac{\beta}{\alpha} g_{v}\right)$ A $g_{v} \equiv 0$, so that the statement of this corollary is exactly Theorem 4.2 applied to $f_{v}+\frac{\beta}{\alpha} g_{v}$ and $g_{v}$ and Corollary 4.3 applied to $f_{v}$ and $g_{v}+\frac{\alpha}{\beta} f_{v}$.

The above result gives us the possibility to give a complete classification of the semi-regular functions whose *-product is slice preserving.

Proposition 4.5. Let $f=f_{0}+f_{v}, g=g_{0}+g_{v} \in \mathcal{R} \mathcal{N}(\Omega) \backslash\{0\}$. Then $f^{\star} g$ belongs to $\mathcal{R} \mathcal{M}_{\mathbb{R}}(\Omega)$ if and only if exactly one of the following holds

1. $f_{v} \equiv g_{v} \equiv 0$;

2. $f_{v} \not \equiv 0$ and $f$ is a multiple of $g^{c}$ for a slice preserving function;

3. $f_{v}$ and $g_{v}$ are linearly independent and $f^{\star} g \equiv 0$.

Case (3) happens only if $\left\langle f_{v}, g_{v}\right\rangle_{\star}=f_{0} g_{0}$ and $f^{s}=g^{s} \equiv 0$. Moreover, if both $f_{0}$ and $g_{0}$ are not identically zero, then $f$ is a linear combination with coefficients in $\mathcal{R \mathcal { M }}_{\mathbb{R}}(\Omega)$ of $g^{c}$ and a zero divisor orthogonal both to 1 and $g_{v}$ (with respect to $\langle,\rangle_{\star}$ ).

Proof. Trivially, if one among (1),(2) and (3) holds, then $f \star g \in \mathcal{R \mathcal { M }}_{\mathbb{R}}(\Omega)$.

Assume now $\left(f^{\star} g\right)_{v} \equiv 0$. If $f_{v}$ and $g_{v}$ are identically zero, then there is nothing to prove. If $f_{v} \equiv 0$ and $g_{v} \not \equiv 0$, then $f^{\star} g=f_{0}{ }^{\star} g=f_{0} g_{0}+f_{0} g_{v}$ which entails that $f_{0} g_{v} \equiv 0$; since $g_{v} \not \equiv 0$, then $f_{0} \equiv 0$ which is a contradiction; the same holds if $g_{v} \equiv 0$ and $f_{v} \not \equiv 0$. If $f_{v}$ and $g_{v}$ are both different from zero and linearly dependent, we can find $\alpha \in \mathcal{R \mathcal { M }}_{\mathbb{R}}(\Omega)$ such that $f_{v}=\alpha g_{v}$. Now $(f \star g)_{v} \equiv f_{0} g_{v}+g_{0} f_{v}=\left(f_{0}+\alpha g_{0}\right) g_{v}$, as $g_{v} \not \equiv 0$, we obtain $f_{0}=-\alpha g_{0}$, which gives $f=-\alpha g_{0}+\alpha g_{v}=-\alpha\left(g_{0}-g_{v}\right)=-\alpha g^{c}$, showing that $f$ is a multiple of $g^{c}$ for a slice preserving function.

Then we are left to deal with the case when $f_{v}$ and $g_{v}$ are linearly independent. The condition $(f \star g)_{v} \equiv 0$ can be written as

$$
g_{0} f_{v}+f_{0} g_{v}+f_{v} \AA g_{v} \equiv 0 .
$$

Comparing this equality with the proof of Corollary 4.4 we obtain that

- $f_{0}^{2}+f_{V}^{s} \equiv 0$, that is $f^{s} \equiv 0$;

- $g_{0}^{2}+g_{v}^{s} \equiv 0$, that is $g^{s} \equiv 0$;

- $\left\langle f_{v}, g_{v}\right\rangle_{\star}=f_{0} g_{0}$, that is $f_{0} g_{0}-\left\langle f_{v}, g_{v}\right\rangle_{\star} \equiv 0$.

Last equality can also be written as $\left(f^{\star} g\right)_{0} \equiv 0$, and together with Formula (4.4), gives $f^{\star} g \equiv 0$. The conclusion of the proof follows again from Corollary 4.4; indeed we can suppose that, up to conjugation by an invertible element in $\mathcal{R} \mathcal{M}(\Omega)$, we have $g_{v}=g_{0} \mathcal{\partial} i$ and $f_{v}=-f_{0} \mathcal{J} i+\lambda \ell^{-, i} \star j$, for a suitable $\lambda \in \mathcal{R} \mathcal{M}_{\mathbb{R}}(\Omega) \backslash\{0\}$. Thus

$$
g=g_{0}(1+\mathcal{J}) \quad \text { and } \quad f=f_{0}-f_{0} \mathcal{\partial} i+\lambda \ell^{-, i} \star j=\frac{f_{0}}{g_{0}} g^{c}+\lambda \ell^{-, i} \star j,
$$

and we are done. 


\section{Embry's Theorem and commutations}

The results contained in the previous section allow us to give a version of Embry's Theorem in the slice (semi-)regular setting. The classical Embry's theorem for linear operators is based on the assumption that the spectra of the operators $A$ and $B$ do not intersect: this condition entails, via the application of SylvesterRosemblum Theorem, that any operator $C$ which commutes with $A+B$ and $A B$ also commutes with $A$ and $B$.

Inspired by [5, Section 4] we give the following definition.

Definition 5.1. Let $f, g \in \mathcal{R} \mathcal{N}(\Omega) \backslash\{0\}$. We say that $f$ and $g$ satisfy Embry's condition if every semi-regular function which commutes with $f+g$ and $f{ }^{\star} g$ also commutes with $f$ and $g$.

Our goal is to discuss under which conditions the functions $f$ and $g$ satisfy Embry's condition. The strategy we adopt is to consider the different cases which can occur for the linear dependence/independence of $f_{v}$ and $g_{v}$. In order to avoid trivialities, in the sequel we always suppose that $f$ and $g$ are not both slice preserving.

We start by considering the case in which one of the two functions $f$ and $g$ is slice preserving and the other is not.

Remark 5.2. If exactly one between $f$ and $g$ is slice preserving, then $f$ and $g$ satisfy Embry's condition. Indeed, we claim that in this case a function $\chi \in \mathcal{R N}(\Omega)$ commutes with $f+g$ and $f{ }^{\star} g$ if and only if it commutes with $f$ and $g$. First of all, up to a rearrangement of $f$ and $g$, we can suppose that $f_{v} \not \equiv 0$ and $g_{v} \equiv 0$. Since $(f+g)_{v}=f_{v}$ and $\left(f^{\star} g\right)_{v}=g_{0} f_{v}$, then $\chi$ commutes with $f+g$ and $f \star g$ if and only if $\chi_{v}$ is a multiple of $f_{v}$ and this last assertion is equivalent to the fact that $\chi$ commutes with $f$. By taking the difference of $f+g$ and $f$, we have that $\chi$ also commutes with $g$, thus showing that $f$ and $g$ satisfy Embry's condition.

We now turn to examine the case when $f_{v}, g_{v} \not \equiv 0$. Following [5, Section 4] we can give an easy sufficient condition on $f$ and $g$ in order they satisfy Embry's condition, namely that the Sylvester operator $\varsigma_{f,-g}$ is an isomorphism.

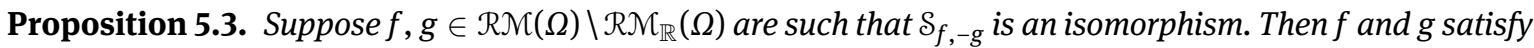
Embry's condition.

Proof. Suppose that $h \in \mathcal{R} \mathcal{N}(\Omega)$ commutes both with $f+g$ and $f{ }^{\star} g$ that is

$$
h h^{\star}(f+g)=(f+g){ }^{\star} h \quad \text { and } \quad h{ }^{\star} f^{\star} g=f^{\star} g{ }^{\star} h .
$$

Premultiplying first equality by $f$, we obtain

$$
f \star h \star f+f \star h \star g=f \star f \star h+f \star g \star h .
$$

Using the second one we get

$$
f \star h \star f+f \star h \star g=f^{\star} f \star h+h{ }^{\star} f \star g,
$$

that is

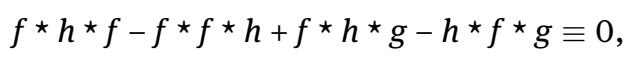

or, equivalently

$$
f \star\left(h \star f-f{ }^{\star} h\right)-\left(h{ }^{\star} f-f{ }^{\star} h\right){ }^{\star} g \equiv 0 .
$$

This means that $h{ }^{\star} f-f{ }^{\star} h$ belongs to the kernel of $\mathcal{S}_{f,-g}$. Since $\mathcal{S}_{f,-g}$ is an isomorphism, this entails that $h \star f-f \star h \equiv 0$, and hence $h$ commutes with $f$; analogously $h$ commutes with $g$ and thus $f$ and $g$ satisfy Embry's condition.

As it is easily seen merely by taking $f \equiv g$, the requirement that $\mathcal{S}_{f,-g}$ is an isomorphism is not necessary in order $f$ and $g$ satisfy Embry's condition, so we deepen our investigation in order to classify exactly which couples of function in $\mathcal{R} \mathcal{N}(\Omega) \backslash \mathcal{R} \mathcal{\mathcal { N } _ { \mathbb { R } }}(\Omega)$ satisfy Embry's condition. 
We begin our analysis by taking into account the case when $f_{v}, g_{v}$ are linearly dependent.

Proposition 5.4. Let $f, g \in \mathcal{R} \mathcal{M}(\Omega) \backslash \mathcal{R} \mathcal{M}_{\mathbb{R}}(\Omega)$ be such that $f_{v}$ and $g_{v}$ are linearly dependent. Then $f$ and $g$ satisfy Embry's condition if and only if $g \neq f^{c}$.

Proof. If $g=f^{c}$, then it is easily seen that $f+g=f+f^{c}=2 f_{0}$ and $f \star g=f \star f^{c}=f^{s}$ both belong to $\mathcal{R} \mathcal{M}_{\mathbb{R}}(\Omega)$, and therefore any function $\chi \in \mathcal{R} \mathcal{N}(\Omega)$ commutes with them. As $f$ is not slice preserving, then there exist functions $\chi \in \mathcal{R} \mathcal{N}(\Omega)$ which do not commute with $f$, and therefore $f$ and $g$ do not satisfy Embry's condition.

Now suppose that $g \neq f^{c}$. Since $f_{v}$ and $g_{v}$ are linearly dependent on $\mathcal{R \mathcal { M }}_{\mathbb{R}}(\Omega)$ and they are both different from zero, then there exists $\alpha \in \mathcal{R \mathcal { M }}_{\mathbb{R}}(\Omega) \backslash\{0\}$ such that $g_{v}=\alpha f_{v}$, thus we can write

$$
(f+g)_{v}=(\alpha+1) f_{v} \text { and }(f \star g)_{v}=g_{0} f_{v}+f_{0} g_{v}=\left(g_{0}+\alpha f_{0}\right) f_{v} \text {. }
$$

As $g \neq f^{c}$, at least one between $\alpha+1$ and $g_{0}+\alpha f_{0}$ is not identically zero, so that $f+g$ and $f \star g$ do not both belong to $\mathcal{R \mathcal { M }}_{\mathbb{R}}(\Omega)$. Take any $\chi \in \mathcal{R} \mathcal{N}(\Omega)$ which commutes with $f+g$ and $f{ }^{\star} g$, the above argument entails that $\chi_{v}$ is an $\mathcal{R \mathcal { M }}_{\mathbb{R}}(\Omega)$-multiple of $f_{v}$. This entails that $\chi$ and $f$ commute, thus showing that $f$ and $g$ satisfy Embry's condition.

The other case to consider is when $f_{v}$ and $g_{v}$ are linearly independent. Next theorem, relying on the last part of the statement of Corollaries 4.3 and 4.4, presents a necessary and sufficient condition, given in terms of the conjugates of $g_{v}$ and $f_{v}$, in order that $f$ and $g$ do not satisfy Embry's condition. In particular, it ensures that when $\Omega$ is slice, then $f$ and $g$ satisfy Embry's condition.

Theorem 5.5. Let $f, g \in \mathcal{R} \mathcal{M}(\Omega)$ be such that $f_{v}$ and $g_{v}$ are linearly independent. Then $f$ and $g$ do not satisfy Embry's condition if and only if one of the following holds

- there exist $h \in \mathcal{R} \mathcal{N}(\Omega)$ invertible, $\lambda \in \mathcal{R \mathcal { M }}_{\mathbb{R}}(\Omega) \backslash\{0\}$ and $\alpha \in \mathcal{R \mathcal { M }}_{\mathbb{R}}(\Omega)$ such that

$$
g_{v}=h^{-\star} \star\left(\left(g_{0}+\alpha\right) \mathcal{J} i\right) \star h \text { and } \quad f_{v}=h^{-\star} \star\left(-\left(f_{0}+\alpha\right) \mathcal{J} i+\lambda \ell^{-, i} \star j\right) \star h ;
$$

- $\quad$ there exist $h \in \mathcal{R} \mathcal{N}(\Omega)$ invertible, $\lambda \in \mathcal{R \mathcal { M }}_{\mathbb{R}}(\Omega) \backslash\{0\}$ such that

$$
g_{v}=h^{-\star} \star \lambda \ell^{-, i} \star j \star h \quad \text { and } \quad f_{v}=-h^{-\star} \star\left(\left(f_{0}-g_{0}\right) \mathcal{J} i\right) \star h .
$$

Proof. As $f_{v}$ and $g_{v}$ are linearly independent, then $(f+g)_{v}=f_{v}+g_{v} \not \equiv 0$.

If $f$ and $g$ do not satisfy Embry's condition, then there exists $\chi \in \mathcal{R} \mathcal{M}(\Omega)$ which commutes with $f+g$ and $f{ }^{\star} g$ and does not commute with $f$ (and $g$ as well). As all elements of $\mathcal{R \mathcal { M }}_{\mathbb{R}}(\Omega)$ commute with any function in $\mathcal{R} \mathcal{M}(\Omega)$, then $\chi$ cannot belong to $\mathcal{R \mathcal { M }}_{\mathbb{R}}(\Omega)$ or equivalently $\chi_{v} \not \equiv 0$. This implies that $f_{v}+g_{v}$ and $(f \star g)_{v}$ are both $\mathcal{R \mathcal { M }}_{\mathbb{R}}(\Omega)$-multiples of $\chi_{v}$ and therefore are linearly dependent on $\mathcal{R \mathcal { M }}_{\mathbb{R}}(\Omega)$. As $(f+g)_{v} \not \equiv 0$, this guarantees that there exists $\alpha \in \mathcal{R \mathcal { M }}_{\mathbb{R}}(\Omega)$ such that $\alpha\left(f_{v}+g_{v}\right)+g_{0} f_{v}+f_{0} g_{v}+f_{v}$ A $g_{v} \equiv 0$, or equivalently

$$
\left(g_{0}+\alpha\right) f_{v}+\left(f_{0}+\alpha\right) g_{v}+f_{v} \wedge g_{v} \equiv 0
$$

We start by considering Equality (5.1) when $g_{0}+\alpha \not \equiv 0$. Since commutation is invariant by conjugating with the same invertible element $h \in \mathcal{R \mathcal { N }}(\Omega)$, thanks to Corollary 4.4, we can suppose that, up to conjugation, we have $g_{v}=\left(g_{0}+\alpha\right) \mathcal{J} i$ and $f_{v}=-\left(f_{0}+\alpha\right) \mathcal{J} i+\lambda \ell^{-, i} \star j$ for a suitable non-vanishing $\lambda \in \mathcal{R \mathcal { M }}_{\mathbb{R}}(\Omega)$; in particular

$$
f=2 f_{0} \ell^{+, i}-\alpha \mathcal{J} i+\lambda \ell^{-, i} \star j \quad \text { and } \quad g=2 g_{0} \ell^{-, i}+\alpha \mathcal{J} i \text {. }
$$

Now we are left to deal with Equality (5.1) when $g_{0}+\alpha \equiv 0$, that is $\alpha=-g_{0}$. As $f_{v}$ and $g_{v}$ are linearly independent, this implies that $f_{0}+\alpha=-\left(f_{0}-g_{0}\right) \not \equiv 0$. Again, since commutation is invariant by conjugating with the same invertible element in $\mathcal{R} \mathcal{N}(\Omega)$, thanks to Corollary 4.3 we can find an invertible $h \in \mathcal{R} \mathcal{N}(\Omega)$ and a non-vanishing $\lambda \in \mathcal{R \mathcal { M }}_{\mathbb{R}}(\Omega)$ such that, up to conjugating by $h$, we have $f_{v}=-\left(f_{0}+\alpha\right) \mathcal{J} i=-\left(f_{0}-g_{0}\right) \mathcal{J} i$ and $g_{v}=\lambda \ell^{-, i} \star j ;$ in particular

$$
f=f_{0}-\left(f_{0}-g_{0}\right) \mathcal{\partial} i=2 f_{0} \ell^{+, i}-g_{0} \mathcal{\partial} i \quad \text { and } \quad g=g_{0}+\lambda \ell^{-, i} \star j .
$$


Vice versa, suppose that, up to conjugation for the same invertible element in $\mathcal{R} \mathcal{N}(\Omega)$, the functions $f_{v}$ and $g_{v}$ can be written as $g_{v}=\left(g_{0}+\alpha\right) \mathcal{J} i \not \equiv 0$ and $f_{v}=-\left(f_{0}+\alpha\right) \mathcal{J} i+\lambda \ell^{-, i} \star j$ for suitable $\lambda \in \mathcal{R \mathcal { M }}_{\mathbb{R}}(\Omega) \backslash\{0\}$ and $\alpha \in \mathcal{R \mathcal { M }}_{\mathbb{R}}(\Omega)$. Then we have

$$
g=2 g_{0} \ell^{-, i}+\alpha \mathcal{J} i \text { and } f=2 f_{0} \ell^{+, i}-\alpha \mathcal{J} i+\lambda \ell^{-, i} \star j
$$

A long but straightforward computation gives that

$$
(f+g)_{v}=\left(g_{0}-f_{0}\right) \mathcal{J} i+\lambda \ell^{-, i} \star j \quad \text { and } \quad(f \star g)_{v}=-\alpha\left(-\left(g_{0}-f_{0}\right) \mathcal{J} i+\lambda \ell^{-, i} \star j\right)
$$

and thus $(f+g)_{v}$ and $\left(f^{\star} g\right)_{v}$ are linearly dependent. In particular, if we take $\chi=f+g$, we have that the function $\chi \in \mathcal{R \mathcal { N }}(\Omega) \backslash \mathcal{R \mathcal { N }}_{\mathbb{R}}(\Omega)$ commutes with both $f+g$ and $f \star g$. As $g_{v}$ is not an $\mathcal{R \mathcal { N }}_{\mathbb{R}}(\Omega)$-multiple of $(f+g)_{v}$, then $g$ does not commute with $\chi$, showing that $f$ and $g$ do not satisfy Embry's condition.

Finally, let us suppose that, up to conjugation for the same invertible element in $\mathcal{R} \mathcal{N}(\Omega)$, the functions $f_{v}$ and $g_{v}$ can be written as $g_{v}=\lambda \ell^{-, i} \star j$ and $f_{v}=-\left(f_{0}-g_{0}\right) \partial i \not \equiv 0$ for a suitable $\lambda \in \mathcal{R} \mathcal{M}_{\mathbb{R}}(\Omega) \backslash\{0\}$. Then we have

$$
g=g_{0}+\lambda \ell^{-, i} \star j \text { and } f=2 f_{0} \ell^{+, i}+g_{0} \partial i
$$

Since

$$
(f+g)_{v}=\left(g_{0}-f_{0}\right) \mathcal{J} i+\lambda \ell^{-, i \star} j \quad \text { and } \quad(f \star g)_{v}=g_{0}\left(\left(g_{0}-f_{0}\right) \mathcal{J} i+\lambda \ell^{-, i} \star j\right),
$$

we have that $(f+g)_{v}$ and $(f \star g)_{v}$ are linearly dependent, while $f_{v}$ and $(f+g)_{v}$ are linearly independent. Reasoning as above, we obtain that $f$ and $g$ do not satisfy Embry's condition, which concludes the proof of the statement.

We finish our investigation on Embry's condition by stating a necessary condition on $f$ and $g$ in order the Embry's condition is not satisfied: the reason why we single out this requirement is that it is easily expressed in terms of $\Omega, f$ and $g$ (and, moreover, it is not far from being sufficient).

Corollary 5.6. Let $f, g \in \mathcal{R} \mathcal{N}(\Omega)$ be such that $f_{v}$ and $g_{v}$ are linearly independent. If $f$ and $g$ do not satisfy Embry's condition, then $\Omega$ is a product domain, $f_{v}^{S}$ and $g_{v}^{S}$ have a square root in $\mathcal{R M N}_{\mathbb{R}}(\Omega)$ and $\left(\left\langle f_{v}, g_{v}\right\rangle_{\star}\right)^{2} \equiv f_{v}^{s} g_{v}^{s}$.

At last, in the case of a slice domain, summarizing up all possible occurrences for $f_{v}$ and $g_{v}$, the following statement gives a very neat necessary and sufficient requirement on $f$ and $g$ in order they satisfy Embry's condition.

Corollary 5.7. Let $f, g \in \mathcal{R} \mathcal{N}(\Omega)$. If the domain $\Omega$ is slice, then $f$ and $g$ satisfy Embry's condition if and only if $g \neq f^{c}$.

\section{References}

[1] A. Altavilla, C. de Fabritiis, *-exponential of slice-regular functions, Proc. Amer. Math. Soc. 147, 2019, 1173-1188.

[2] A. Altavilla, C. de Fabritiis, s-Regular functions which preserve a complex slice, Ann. Mat. Pura Appl. (4) 197:4, 2018, 12691294.

[3] A. Altavilla, C. de Fabritiis, Equivalence of slice semi-regular functions via Sylvester operators, preprint arXiv:1907.07385.

[4] A. Altavilla and G. Sarfatti. Slice-Polynomial Functions and Twistor Geometry of Ruled Surfaces in $\mathbb{C P}^{3}$. Math. Z. 291(3-4) (2019), 1059-1092.

[5] R. Bhatia, P. Rosenthal, How and why to solve the operator equation $A X-X B=Y$ ? Bull. London Math. Soc. 29 (1997) 1-21.

[6] V. Bolotnikov, Polynomial interpolation over quaternions, J. Math. Anal. Appl., 421(1), 2015, 567-590.

[7] V. Bolotnikov, On the Sylvester Equation over Quaternions, Operator Theory: Advances and Applications, Volume 252, (2016), Pages 43-75.

[8] F. Colombo, J. Oscar Gonzalez-Cervantes, I. Sabadini, The C-property for slice regular functions and applications to the Bergman space, Compl. Var. Ell. Eq., 58, n. 10 (2013), 1355-1372.

[9] G. Gentili, C. Stoppato, D. C. Struppa, Regular Functions of a Quaternionic Variable, Springer Monographs in Matehmatics, Springer, 2013. 
[10] R. Ghiloni, V. Moretti, A. Perotti, Continuous Slice Functional Calculus in Quaternionic Hilbert Spaces, Rev. Math. Phys. 25 (2013), 1350006-1-1350006-83.

[11] R. Ghiloni, A. Perotti, C. Stoppato, The algebra of slice functions, Trans. of Amer. Math. Soc., Volume 369, N.7, 2017, pp.47254762.

[12] R. Ghiloni, A. Perotti, and C. Stoppato. Singularities of slice regular functions over real alternative *-algebras. Adv. Math., 305:1085-1130, 2017.

[13] R. Ghiloni, A. Perotti, C. Stoppato, Division algebras of slice functions, to appear in Proceedings A of the Royal Society of Edinburgh DOI:10.1017/prm.2019.13.

[14] J. Sylvester, Sur l'equations en matrices $p x=x q$, C.R. Acad. Sci. Paris 99 (1884) 67-71, 115-116. 